\title{
Entre el discurso y la práctica en educación y pedagogía social ${ }^{*}$
}

\author{
Between speech and practice in education and social pedagogy
}

\section{Resumen}

Con el fin de verificar el estado de los atributos de la Educación y de la Pedagogía Social en la Licenciatura en Educación Básica, con énfasis en Educación Artística de la Fundación Universitaria Católica lumen Gentium, el Semillero de investigación Educación Artística en plural desarrolló el Proyecto de investigación titulado Discursos y Prácticas en Educación y Pedagogía Social en la Licenciatura en Educación Básica con énfasis en Educación Artística de la Fundación Universitaria Católica Lumen Gentium en el año 2016. Con un enfoque metodológico mixto por el que se construyeron dos instrumentos para la recolección de datos, uno cualitativo y el otro cuantitativo. Estos se aplicaron a los docentes, directivas y estudiantes de la Licenciatura respectivamente.

Los resultados obtenidos permiten comprender la coherencia entre el discurso y la práctica, tanto de los docentes como de los estudiantes res pecto a los atributos de la educación y Pedagogía Social que se identificaron como: diálogo entre maestro y educando; diálogo entre el sujeto y su contexto local, nacional y global y propuesta de acción del sujeto en la misma sociedad.

Por lo cual se concluye que la Licenciatura estudiada debe construir estrategias y herramientas que posibiliten la coherencia entre la praxis y la teoría. Así como también, cuestiona el lugar que tiene la educación artística dentro de los procesos que se agencian en la Educación y Pedagogía Social.

Palabras clave: Discursos y prácticas, educación y pedagogía social, educación artística, Licenciatura en Educación Básica con énfasis en Educación Artística, Fundación Universitaria Católica Lumen Gentium.

\footnotetext{
* Texto realizado como informe del Proyecto de Investigación Discursos y Prácticas en Educación y Pedagogía Social en la Licenciatura en Educación Básica con énfasis en Educación Artística de la Fundación Universitaria Católica Lumen Gentium en el año 2016, desarrollado por el Semillero de Investigación Educación artística en plural vinculado al Grupo de Investigación EDUCARTE. Educaciones, Artes y Tecnologías de la Fundación Universitaria Católica Lumen Gentium.

* Maestra en Artes Plásticas y Especialista en Educación Artística Integral de la Universidad Nacional de Colombia, Docente de la Licenciatura en Educación Básica con énfasis en Educación Artística de la Fundación Universitaria Católica Lumen Gentium, Líder del Semillero de Investigación Educación artística en plural vinculado al Grupo de Investigación EDUCARTE. Educaciones, Artes y Tecnologías, Correo electrónico bpacheco@unicatolica.edu.co
} 


\section{Abstract}

In order to see the state of the attributes of Education and Social Pedagogy in the Licenciatura en Educación Básica con énfasis en Educación Artística of the Fundación Universitaria Católica Lumen Gentium, the Semillero de investigación Educación Artística en plural developed the project entitled Discourses and Practices in Education and Social Pedagogy in the Degree in Licenciatura en Educación Básica con énfasis en Educación Artística of the Fundación Universitaria Católica Lumen Gentium in the year 2016, with a mixed methodological approach by which two instruments were built for the collection of data, one qualitative and the other quantitative. These applied to teachers, directives and students respectively.

The results obtained allow to understand the coherence between the discourse and the practice of both, teachers and students, regarding the attributes of education and social pedagogy that is identified as: dialogue between teacher and apprentice; dialogue between the subject and the local, national al global context; and proposal of action of the subject in the society itself.

Therefore, it is concluded that the studied Program must have strategies and tools that allow coherence between praxis and theory. As well as it questions the place that has the artistic education within the processes that are used in the Education and the Social Pedagogy.

Keywords: Discourses and practices. education and social pedagogy, Artistic education, Licenciatura en Educación Básica con énfasis en Educación Artística, Fundación Universitaria Católica Lumen Gentium.

\section{Introducción}

El artículo es producto del informe del Proyecto Discursos y Prácticas en Educación y Pedagogía
Social en la Licenciatura en Educación Básica con énfasis en Educación Artística de la fundación Universitaria Católica lumen Gentium, en el año 2016, que se llevó a cabo por el Semillero de investigación Educación Artística en plural como un ejercicio de acercamiento a la realidad universitaria. Tenía como principal objetivo verificar cómo sucedían los atributos de la pedagogía social en la Licenciatura, ya que esta cuenta con un número significativo de estudiantes que están vinculados a diferentes propuestas de la Educación No Formal, proporcionándoles conocimientos específicos sobre la educación y pedagogía social.

Por tanto, se consideró que los atributos de la Educación y de la Pedagogía Social tienen un eje central que se basa en el ejercicio dialógico, desde aquí se comprende cómo debe establecerse la relación entre maestro y educando, y la relación del sujeto con su entorno local, nacional y global que, a su vez, debe contemplar los aspectos sociales, económicos y políticos.

Como ha de saberse, en todo diálogo es indispensable la interacción de los dos actores que intervienen en la acción. En el caso de la educación y la pedagogía social, luego de que el educando ha logrado realizar una lectura crítica de la realidad, se espera que este se haga consciente de su rol dentro de la misma para que sea él quien propone y agencie los procesos necesarios para la transformación de esta realidad.

\section{Metodología y materiales}

Por tanto, el objetivo del proyecto se centró en revisar cómo sucedían los atributos de la educación y pedagogía social en la Licenciatura en Educación Básica con énfasis en Educación Artística (LEBEA) de la Fundación Universitaria Católica Lumen Gentium en el año 2016, entonces, la muestra seleccionada consistió en el estudiantado de LEBEA y en el personal docente y administrativo que proporcionara información desde las 
cátedras y prácticas académicas vinculadas al desarrollo social o comunitario de los estudiantes.

En los primeros, se concretó un censo a los estudiantes con el fin de definir los lazos que tienen, o que han tenido, estos con procesos comunitarios. De la misma manera, determinar el papel de Unicatólica como una institución académica que ha de capacitarlos para este tipo de labores con la sociedad. En los segundos, se comprendió que los docentes y directivas definen y trasmiten el conocimiento conforme a sus propias experiencias y expectativas en la docencia y la pedagogía.

De este modo, se diseñaron dos tipos de instrumentos que se aplicarían a las muestras seleccionadas: a los estudiantes se les aplicó una encuesta con metodología CAP (Conocimientos, Actitudes y Prácticas), que permitirían revisar con profundidad la ruta de los conocimientos en educación y pedagogía social en ellos. Las preguntas de caracterización, ayudaron a definir, en términos sociales, laborales y económicos, el lugar desde el cual se ubicaban los estudiantes con respecto al objetivo planteado por el Proyecto. En cuanto a las preguntas para revisar los conocimientos, las actitudes y las prácticas de los estudiantes, se plantearon como preguntas de selección múltiple y cada una de las respuestas permitió comprender a qué vertiente de la educación y pedagogía social se inclinaba cada uno de los estudiantes. Las preguntas destinadas a revisar los conocimientos, dieron cuenta de lo que los estudiantes han entendido respecto al tema desde su experiencia. Las preguntas para las actitudes se organizaron como preguntas de rango de importancia, con el propósito de identificar los motivos que tienen los estudiantes al realizar sus ejercicios docentes. Por último, las preguntas destinadas a indagar por las prácticas se realizaron como tipo de preguntas de acción.

El segundo instrumento, las entrevistas, se diseñaron semiestructuradas. Las preguntas indagaron, principalmente, por la experiencia en relación con el ejercicio docente y pedagógico de cada uno de los entrevistados así: el primer contacto y acercamiento a la docencia, vinculación con Unicatólica y el ejercicio actual como profesor de esta y la conclusión que el profesor estima de su trabajo.

Las entrevistas se realizaron a docentes, directivas y a algunos estudiantes que habían mostrado tener vinculación con el ámbito no formal en educación.

De esta manera, se diseñaron los instrumentos que, a su vez, dieron cuenta de su articulación con el marco teórico planteado para el presente proyecto, en el que se definieron los conceptos discurso, práctica pedagógica y pedagogía social, este último con sus cuatro vertientes: educación especializada, educación de adultos mayores, animación sociocultural y pedagogía social (Rubio, 2015).

\section{Discursos y prácticas pedagógicas}

El concepto definido por Alvin Gouldner (1978) para discurso, permitió plantear las preguntas de la entrevista basadas en la experiencia, puesto que es desde aquí que el sujeto reflexiona y dimensiona su acción, tanto para dar una justificación a las acciones realizadas, como para direccionar las futuras. De la misma manera, se puede comprender el discurso que desarrollan los estudiantes, a través de las respuestas dadas en las preguntas enfocadas a las prácticas y actitudes.

En cuanto al término práctica pedagógica, se relacionó con la mayoría de las preguntas dentro de los dos instrumentos: en las entrevistas estuvieron de manera implícita y en las encuestas se desarrolló dándole al estudiante la opción de informar su actividad docente dentro de cada uno de los ámbitos educativos formal y no formal.

Aunque la pedagogía social exige un tipo de discurso político claro y completamente depen- 
diente del ejercicio práctico- conforme a lo planteado por Giroux (2001)-, para el presente proyecto se contempló la posibilidad de revisar las relaciones entre los discursos y las prácticas en educación y pedagogía social, tanto del estudiantado como de los docentes y directivas, de tener algunas incoherencias, ya que es posible que haya diferencias o contradicciones entre lo uno y lo otro, permitiendo lecturas que dan cuenta del proceso de construcción de la actividad pedagógica en los sujetos de la muestra.

A continuación se muestran los resultados que se comprendieron a partir del análisis de los datos obtenidos.

\section{Resultados}

\section{Relación dialógica entre maestro y educando}

Conforme al ejercicio dialéctico propio de la pedagogía y la educación social, se relaciona el rol que desarrollan el maestro y el educando: En el aspecto práctico, las encuestas aplicadas a los estudiantes permiten ver que un gran porcentaje de los estudiantes amplían en sus clases la participación de sus educandos, ya sea en las decisiones de las temáticas, didácticas o evaluaciones a abordar en el programa de curso, tanto para el caso de la Educación Formal como de la No Formal.

De acuerdo a lo planteado por García Molina (2003), la pedagogía social debe promover la educación para la vida pública. En este sentido, el ejercicio de involucrar a los educandos en la toma de decisiones tiene que ver con uno de los principales cometidos de la Pedagogía y Educación Social, en la vertiente de la animación sociocultural, a la hora de lograr un sujeto que se comprenda a sí mismo como un sujeto activo dentro de su comunidad.

Igualmente, las respuestas entregadas por los estudiantes en cuanto al aspecto actitudinal y al cognitivo; ambas, reflejan una postura de acuerdo a la vertiente animación sociocultural, ya que, en el primer aspecto, un poco más de la mitad del estudiantado (53 \%) considera la acción del maestro como un gestor de capacidades y la del educando, como un sujeto activo dentro de su comunidad. Así mismo sucede en el segundo aspecto, los estudiantes (48 \%) que señalan a manera de definición al ideal de sujeto de la educación popular como el sujeto activo, incluyente y justo que, aunque no sea esta la respuesta correcta, ya que la definición pertenece a animación sociocultural, da cuenta de lo que han aprendido sobre la educación y pedagogía social.

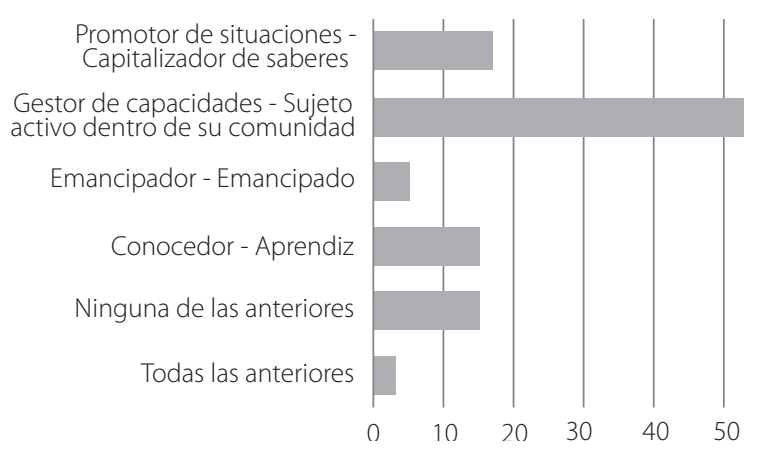

Gráfico 1. Un sinónimo para profesor y otro para estudiante, Licenciatura Básica con énfasis en Educación Artística en la Fundación Universitaria Católica Lumen Gentium Cali.

Fuente: Encuesta Proyecto Discursos y prácticas académicas en educación y pedagogía social en LEBEA, Unicatólica, 2016.

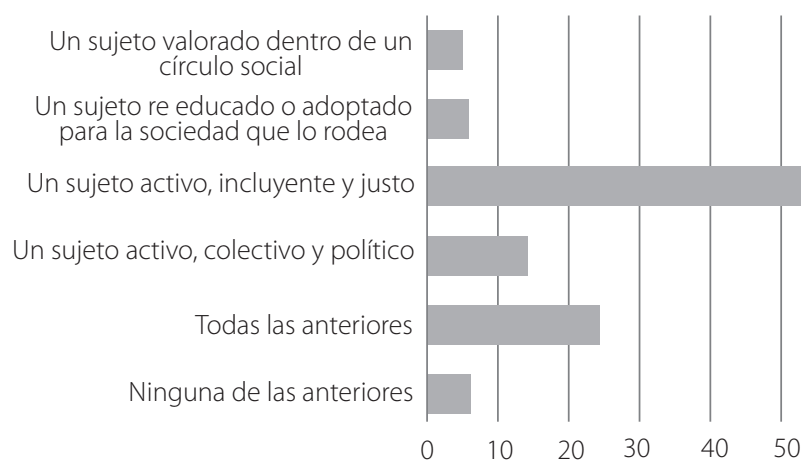

Gráfico 2. Ideal de sujeto al que desea construir la educación popular. Licenciatura Básica con énfasis en Educación Artística en la Fundación Universitaria Católica Lumen Gentium Cali.

Fuente: Encuesta Proyecto Discursos y prácticas académicas en educación y pedagogía social en LEBEA, Unicatólica, 2016. 
Por parte de las directivas y docentes, cada cual tiene un enfoque hacía una de las vertientes de manera independiente, que oscilan entre la animación sociocultural y la pedagogía social, en el caso de los docentes que han logrado algún tipo de estudios relacionado con la pedagogía, ya sea como pregrado o como un curso de extensión que les permita tener las competencias adecuadas para la labor docente. En el caso de los docentes que no han tenido este desarrollo, se nota un discurso que ha construido los contenidos en miras a un pensamiento crítico, pero que aún no se ha logrado vincular a la práctica como ejercicio pedagógico. Un ejemplo de esto se evidencia en la pregunta realizada a los docentes sobre las estrategias o metodologías empleadas para la ejecución de sus clases, en la que dan cuenta de la actitud que conciben en el rol de maestro-educando:

El Director de LEBEA respondió lo siguiente:

“Bueno, referentes. Bueno, como te digo. La historia del arte como tal, la conocida o la que se está escribiendo actualmente con los nuevos hallazgos, que es un poco más interesante, genera ese ejercicio crítico; lo metodológico es a través de las TICS, lo que trato es de generar, de utilizar mucho la web, las cosas que yo enuncio en clase siempre las pongo en contra colación o siempre les digo: miren, esto es lo que piensa tal persona, entonces la metodología es muy abierta a no ser una enseñanza solo, digamos, como un cierto monólogo, sino que siempre yo interactúo mucho con los estudiantes en el sentido que ellos puedan, también, el enunciado mío ponerlo en cuestión. Esos cuestionamientos me interesan mucho, creo que se les da también un aire de libertad y de maniobra al estudiante frente al curso y eso los motiva a seguir viniendo" (A. Buzzi, comunicación personal, 22 de agosto de 2016).

La Directora del Área de Humanidades respondió a la misma pregunta:

"Aparte de la teoría general, beneficio de teoría general, trato de buscar análisis comparativos estos también funcionan mucho y la estrategia a través de casos particulares y el juego de roles, esto lo aprendí en derecho pero creo que se aplica muy bien a otros temas en particular" (V. Betancourt, comunicación personal, 18 de agosto de 2016).

El Licenciado a cargo de las Prácticas Pedagógicas de LEBEA, respondió:

"Cómo hago ya una clase. Ahora estoy completamente convencido que la comunicación con el estudiante es fundamental. Establecer unos buenos vínculos, unos buenos mecanismos de comunicación con los estudiantes, son fundamentales; entonces, siempre trato de abrir ese espacio para el diálogo, para escucharlos. El primer momento en la primera clase, es lo que yo hago: abrir un espacio en el que ellos comuniquen, expresen sus inquietudes, qué expectativas tienen, de dónde vienen y a dónde quieren ir, qué esperan a partir de esa experiencia. Y no hacemos unos acuerdos, pero yo sí tengo en cuenta esas consideraciones y trato de incluirlas, de alguna manera, en el contenido que yo tengo planeado" (W. Sánchez, comunicación personal, 12 de septiembre de 2016).

Con lo anterior, se puede concluir que hay tres tipos de ejecución del rol de maestro - educando y la acción que construye la relación entre estos. La primera tiene que ver que con la propuesta expositiva, en donde el docente enuncia unos juicios que revisan el contexto local, nacional o global de manera crítica como un ejercicio de lectura. En la segunda, esta lectura se articula con ejercicios de didáctica que permiten que los estudiantes desarrollen competencias de análisis crítico. La tercera, se propone como un ejercicio cotidiano donde la voz del estudiante es importante dentro de la construcción de la clase.

Se debe resaltar que, de la misma manera, hay diversidad en las formas de cómo fundar este ejercicio dialéctico, para algunos de los docentes ha de cumplir con la construcción de conocimiento sin confundirse con que, dentro de este proceso, se borren los límites de los roles entre maestro y educando. Así lo plantea la docente del Área de Proyección social, al preguntarle sobre la importancia en la diferenciación de los roles. 
“[...] porque siempre trato de ponerlos en un lugar de diferenciación hacia mí, más cuando tengo estudiantes que son mayores que yo, pero que sepan que lo que vamos a hacer es una construcción de conocimiento conjunto y aunque nuestras posiciones sean diferentes, eso no significa que no podamos hacer esa construcción, no se necesita que seamos iguales, que en eso sí se puede pecar en eso de construir con el otro, todos somos seres humanos, pero cuando estamos en un espacio que nos ubica en dos roles no somos iguales y esos dos roles deben tener su funcionalidad. Entonces, si tú eres estudiante tienes unas funciones, si soy docente yo tengo otras" (G. García, Comunicación personal, 10 de octubre de 2016).

Para el Líder de las Prácticas Pedagógicas de LEBEA, se concibe ese espacio de intercambio de otra forma, al preguntarle por el primer encuentro entre maestro-educando, indica que es importante borrar las barreras:

"Primero me salgo del escritorio y me siento en las mismas sillas que ellos, me acerco a ellos, entro en el grupo, eso es lo primero y trato de no tener, de quitarme de encima el rótulo de docente; entonces trato de desaparecer la distancia que existe entre las dos partes en ese momento. Hay que dinamizar, de alguna manera, ese diálogo para que ellos, en la medida que uno va hablando, vean que hay cosas que les toca y sobre ese tema me apoyo para motivar la respuesta de ellos. Ahora, cómo es la clase en sí, el primer momento por supuesto es el saludo, pero es un saludo que no es para nada autoritario, porque yo vengo de una escuela donde el modelo era ese conductista, un docente autoritario" (W. Sánchez, comunicación personal, 12 de septiembre de 2016).

Se entiende esta diversidad de acciones que definen el rol de maestro-educando para establecerlo dentro del proceso dialógico como el método -conforme a lo planteado por Freire- ya que este debe evidenciar la exteriorización de la conciencia y materializarse en actos.

\section{La relación dialógica entre sujeto y contexto}

En esta misma dirección dialógica se plantea la relación que existe entre el individuo y su contexto social local, nacional y global. Es decir, lo que le permite al sujeto hacer una lectura crítica de la realidad.

La encuesta aplicada a los estudiantes permite señalar que, en cuanto a los aspectos prácticos, se evidenció una diferencia mínima en dos acciones que en el ámbito no formal se desarrollan más fuertemente, estas tienen que ver con las temáticas que se articulan con el contexto inmediato de la comunidad académica -si las problemáticas locales, nacionales y globales son tenidas en cuenta; y, de la misma manera, si la práctica pedagógica responde a estas problemáticas-. El que los maestros en el ámbito formal las no las lleven con suficiente frecuencia a cabo, se puede entender como una limitante propuesta desde las mismas instituciones formales, ya que los contenidos del plan de aula de cada asignatura exigen el cumplimiento de estos. Por lo que cabe preguntarse, en este sentido, por la real articulación de los contenidos transversales propuestos por el Ministerio de Educación que pretende que las problemáticas de índole ecológica, social y política se abarquen en diversas asignaturas sin modificar sus contenidos, ya que se pueden proponer como temas de discusión dentro de los mismos argumentos de asignatura. Dicho de este modo, no parece tan viable que estos temas transversales se logren dar de la manera más pertinente $y$, al mismo tiempo, cumplir con los contenidos de cada asignatura.

En cuanto a los dos postulados en los que las prácticas en espacios educativos no formales superaron las prácticas que se inscriben en lo formal, dan cuenta de lo que Freire denominó "lectura crítica de la realidad" y permiten que el sujeto se piense a sí mismo como un sujeto político en su discurso y en su accionar. Es decir, que tiene que ver con la vertiente de la Educación Popular. Esta a su vez, contempla lo que Cambi (2005), define para la pedagogía social como una propuesta que debe responder a las necesidades de educación de las socie- 
dades, considerando que es en esta lectura crítica de la realidad donde al sujeto se le hace partícipe de las necesidades de su propia comunidad. Lo anterior confirma lo planteado por Giroux (2001), en tanto que, la pedagogía social debe supeditar las prácticas a los distintos contextos en los que se desarrolle.

En el aspecto actitudinal, las respuestas dadas a la pregunta: “¿Según usted cómo sería una clase ideal?" Permiten ver que una mayoría de los estudiantes $(40 \%)$ consideran que el intercambio que ha de construirse entre educando y su contexto, se enmarca en un diálogo de saberes entre los participantes del aula de clase. Sin embargo, parece no tener mayor importancia para los procesos dentro de esta, el que los educandos se hagan conscientes de su rol de acción dentro de la sociedad.

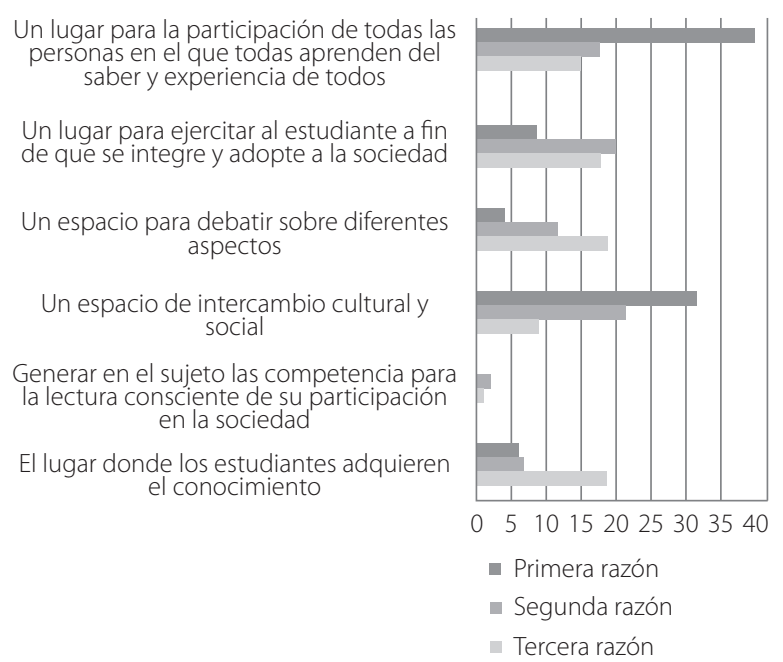

Gráfico 3. Concepto de «clase» ideal. Licenciatura Básica con énfasis en Educación Artística en la Fundación Universitaria Católica Lumen Gentium Cali.

Fuente: Encuesta Proyecto Discursos y prácticas académicas en educación y pedagogía social en LEBEA, Unicatólica, 2016.

En el aspecto cognitivo, el $48 \%$ de los estudiantes comprenden que en la educación y pedagogía social, la relación del individuo debe establecerse con el contexto que le rodea en términos que van más allá de las necesidades individuales del sujeto y que debe ampliarse hasta llegar a los ámbitos sociales, económicos y políticos que afectan a las poblaciones.
Por otra parte, en las entrevistas realizadas a los docentes, la Líder de las Prácticas Pedagógicas de la Facultad de Educación, ante la pregunta: “Freire también dice que es importante que el profesor sea crítico y que por ende también debe formar a sus educandos de manera crítica. ¿Cómo se logra hacer eso?", responde:

"Pues la apuesta es muy grande, la apuesta es muy grande porque nosotros tenemos un sistema educativo perverso. O sea, tenemos un sistema que no invita a nada, que invita a reproducir, que invita a estar enajenado todo el tiempo, invita es a estar preocupado por otras cosas, a olvidarnos de lo humano, es poco humanizante este sistema educativo que nosotros tenemos, pero en los estudiantes hay que impactar con cosas de su humanidad y uno se vuelve crítico y puede lograr transformaciones y cambios de actitud de ellos con cosas tan sencillas como el ejemplo, con cosas tan sencillas como... como reflexiones muy puntuales, eso de hablarles de una noticia que ellos vieron de algo que pasó en el barrio o de algo que sucedió en la escuela, entonces está en los pequeños detalles. Uno no puede creer que porque nos gusta o tenemos la línea de la pedagogía crítica entonces que, de un momento para otro, vamos a cambiar las cosas, no. Uno sabe que eso no es así tan sencillo, pero que uno o dos o tres estudiantes ya se motiven en participar de un proyecto [...], o que ya le indaguen a uno, o que vayan y lo busquen a la sala de profesores y le digan: profesora venga denos clase para que hablemos del tema último que estamos hablando. Allí estamos haciendo un ejercicio y es la invitación y es la apuesta que yo siempre les digo a los que se están formando como futuros licenciados o como futuros docentes. O sea, si nosotros estamos carentes de crítica entonces no vamos a poder transformar nada y vamos a seguir reproduciendo lo que hicieron con nosotros que son clases lineales, clases totalmente verticales, clases donde no hay coherencia, clases donde solamente hay una información, pero pues, como no nos importa, entonces, simplemente, pues vamos y damos nuestro discurso, recibimos un cheque de un sueldo y no transformamos absolutamente nada. Entonces digamos que ese es el ejercicio de lo que uno plantea y también lo dice Freire es muy interesante. Hay un ejercicio y una apues- 
ta muy grande frente a la lectura y la escritura, si nosotros no leemos somos malos escritores y uno debería inculcar mucho más en la escuela el ejercicio de la lectura, pero la lectura consiente y en la universidad también y es una invitación todo el tiempo, que por eso es la otra apuesta que nosotros tenemos además de las prácticas que hacemos con el Encuentro de Experiencias Significativas y que hacemos también a través del ejercicio de la revista. Es decir, invitamos a los estudiantes que todos esos elementos tan positivos y todas esas fortalezas que ellos tienen, las puedan mostrar y las puedan representar en esos dos ejercicios que son tan válidos, que son el de la Revista y las Experiencias, pero, allí uno se encuentra mucha pereza frente al ejercicio de la lectura y el maestro tiene eso y es una reflexión que yo hago con ellos y es que parece ser que cuando el maestro ya está en el escenario de la escuela y ya hay una apuesta educativa, hay tanta confianza en lo que se hace que no permite que entren nuevos procesos ¿no?, o uno insertarse a nuevos procesos, o uno como que se cierra y no permite escuchar al otro, entonces yo digo que eso sí es un error de nosotros y que me parece que es lo bonito de la pedagogía social, el ejercicio de la escucha es muy importante, o sea, el ejercicio de la escucha y uno reflexionar a partir de lo que escucha, entonces, para ser críticos fundamentalmente conocer el contexto, el contexto determina eso pero, ante todo que uno quiera actuar, porque si uno no actúa pues uno puede quedarse en el ejercicio crítico, ¿no?" (P. Quiceno, comunicación personal, 22 de agosto de 2016).

\section{El sujeto transformador}

A la pregunta realizada al Director de LEBEA: ¿Qué espera de un egresado?, responde:

"Primero, que tenga un pensamiento crítico frente a una sociedad, frente a un sistema y frente a la educación como tal, que sea investigativo, que proponga, que aproveche lo creativo y lo expresivo del arte, o sus conocimientos en el arte, para fomentar nuevos entornos, nuevas dinámicas, nuevas didácticas si se quiere, o nuevas propuestas investigativas que puedan servir como fundamento o suministros para sugerir o plantear nuevas alternativas en la educación artística a nivel nacional" (A. Buzzi, comunicación personal, 22 de agosto de 2016).

Como puede observarse el ejercicio educativo conlleva en sí las expectativas sobre el sujeto ideal que se espera lograr mediante el acto educativo, de cómo debe de vincularse con la sociedad este sujeto. En este sentido se realizaron ciertas preguntas a los estudiantes en el aspecto actitudinal:

La primera es: ¿Qué impactos busca -o buscaría- generar en estudiantes con el ejercicio de la pedagogía?: el 49 \% de los estudiantes dieron el primer rango de importancia a la respuesta: Apropiación de los contenidos.

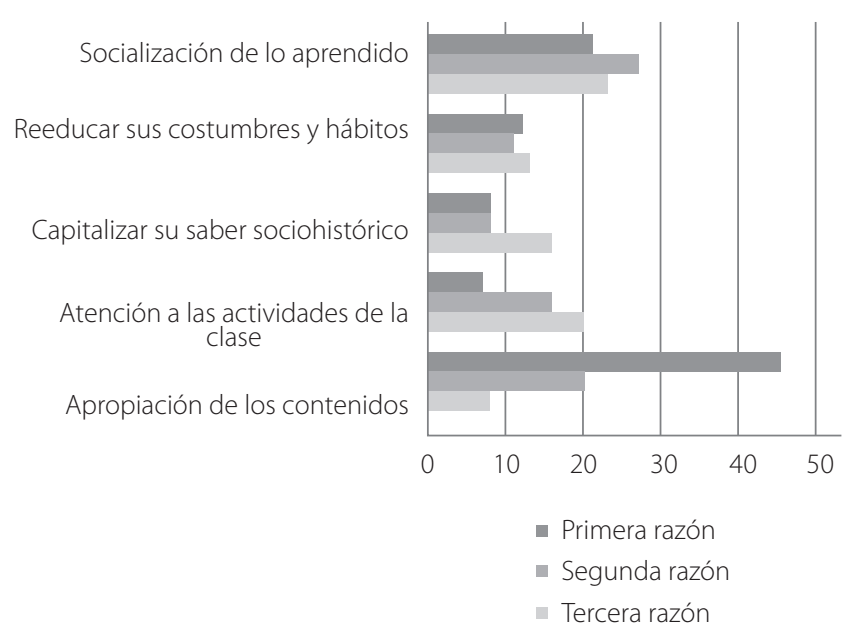

Gráfico 4. ¿Qué impactos busca -o buscaría- generar en estudiantes con el ejercicio de la pedagogía? Licenciatura Básica con énfasis en Educación Artística en la Fundación Universitaria Católica Lumen Gentium Cali.

Fuente: Encuesta Proyecto Discursos y prácticas académicas en educación y pedagogía social en LEBEA, Unicatólica, 2016

La segunda, ¿cuál debería ser el objetivo ideal de la educación?: un 32 \% de los estudiantes ubicó la respuesta: Propiciar desde la revisión del contexto del individuo la transformación de la sociedad, como la primera opción en el rango de importancia. 


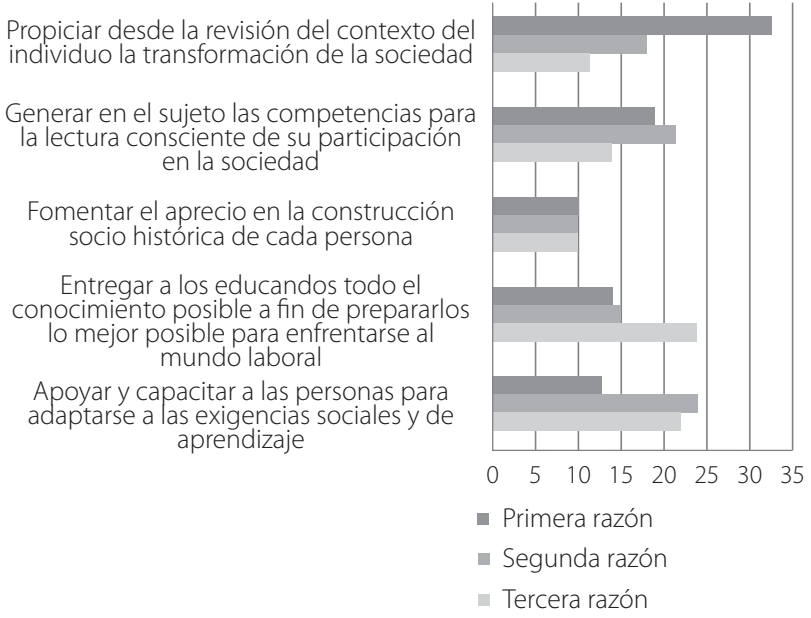

Gráfico 5. Objetivo ideal la educación Licenciatura Básica con énfasis en Educación Artística en la Fundación Universitaria Católica Lumen Gentium Cali.

Fuente: Encuesta Proyecto Discursos y prácticas académicas en educación y pedagogía social en LEBEA, Unicatólica, 2016.

La tercera: ¿Qué impactos busca -o buscaría- generar en estudiantes con las prácticas artísticas?, el $30 \%$ de los estudiantes indicó en primer lugar de importancia, la respuesta: Generar capacidades críticas y de transformación ante problemáticas vividas, transmitir valores y principios para orientarse en el mundo.

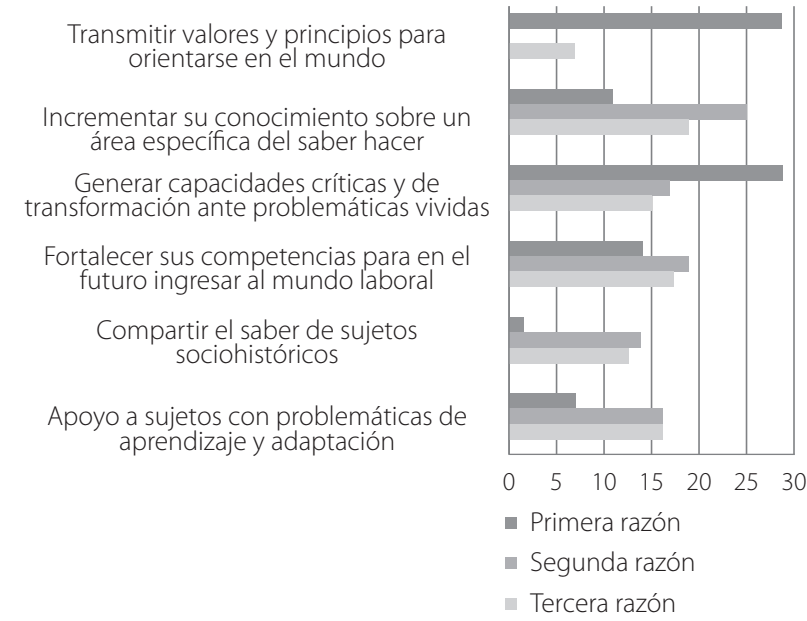

Gráfico 6. ¿Qué impactos busca -o buscaría- generar en estudiantes con las prácticas artísticas? Licenciatura Básica con énfasis en Educación Artística en la Fundación Universitaria Católica Lumen Gentium Cali.

Fuente: Fuente: Encuesta Proyecto Discursos y prácticas académicas en educación y pedagogía social en LEBEA, Unicatólica, 2016.

\section{El lugar del arte o de la educación artística dentro de la pedagogía social}

En los instrumentos elaborados se plantearon preguntas que abordaron directamente el tema de la educación artística. El 31 \% de los estudiantes que laboran en instituciones de Educación Formal registraron que entre sus prácticas evalúan a menudo conocimientos, normas, valores y costumbres artísticas, históricamente establecidas de manera precisa y el $18 \%$ lo realiza siempre. En contraste con las prácticas que desarrollan los estudiantes que trabajan en Educación No Formal, el 18 \% lo realiza a menudo y el $10 \%$, siempre. En la pregunta está de manera implícita una definición de arte y de educación artística que más adelante se contradice con las definiciones que los estudiantes aportan, sobre todo, en el caso del primer grupo.

Según esta pregunta, el arte es una construcción con normas, valores y costumbres establecidas históricamente, lo que puede ser admitido para valorar las prácticas culturales o las propuestas disciplinares de los diferentes pueblos. En este mismo sentido, la misma pregunta indica que la educación artística es una propuesta que exige precisión en el saber y en el hacer.

Sin embargo, a la hora de definir el arte, el $35 \%$ del total de los estudiantes lo definieron como herramientas cognitivas que preparan a los alumnos para la vida: entre otras el desarrollo de habilidades como el análisis, la reflexión, el juicio crítico y, en general, lo que denominamos el pensamiento holístico y el $33 \%$ de los estudiantes señalaron que el objetivo de la educación artística es contribuir a estimular la creatividad, las prácticas culturales y al desarrollo de los territorios. Las definiciones de arte y educación artística señaladas, revelan al ejercicio artístico como un proceso más que como un instrumento ya dado. 


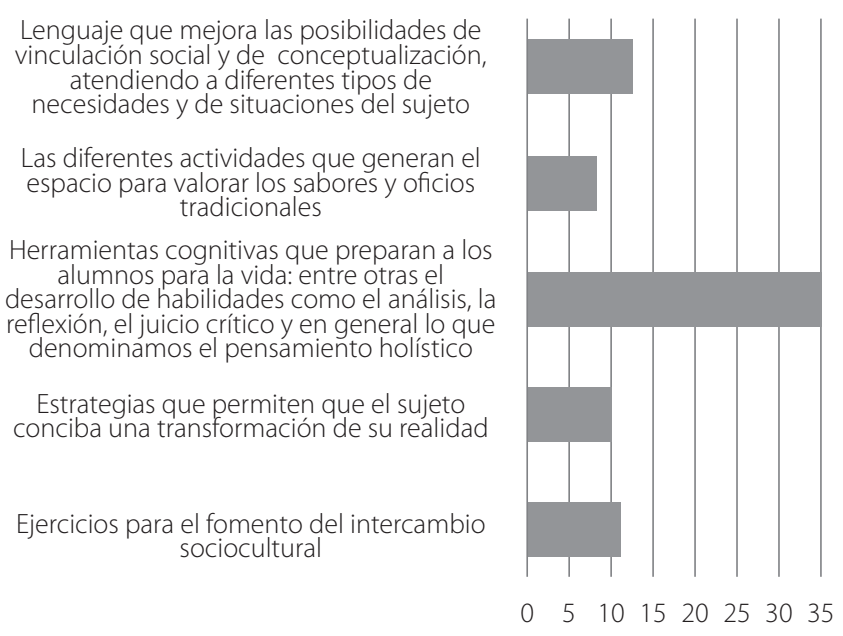

Gráfico 7. Definición de arte dentro de la Educación artística. Licenciatura Básica con énfasis en Educación Artística en la Fundación Universitaria Católica Lumen Gentium Cali.

Fuente: Fuente: Encuesta Proyecto Discursos y prácticas académicas en educación y pedagogía social en LEBEA, Unicatólica, 2016.

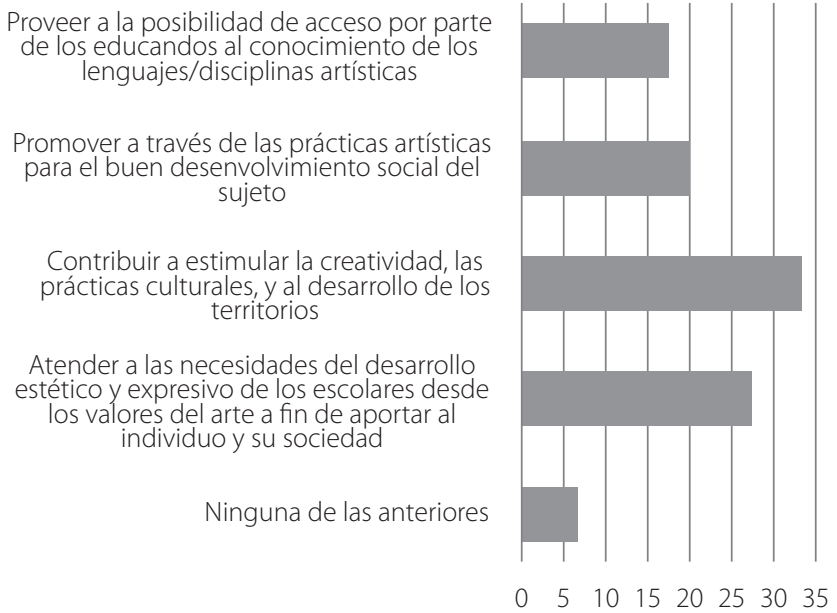

Gráfico 8. Objetivo de la educación artística: Licenciatura Básica con énfasis en Educación Artística en la Fundación Universitaria Católica Lumen Gentium Cali.

Fuente: Encuesta Proyecto Discursos y prácticas académicas en educación y pedagogía social en LEBEA, Unicatólica, 2016.

Es decir, los estudiantes aún no tienen claridad entre la coherencia que debe de haber entre el concepto de arte, educación artística y práctica de la educación artística.

Por último, en las entrevistas que se realizaron tanto a algunos estudiantes como los docentes y direc- tivas, se plantearon preguntas que permiten revisar cómo se desarrolla el ejercicio de la educación artística y el concepto que dentro de estas se establece de educación artística y de arte.

Un estudiante que desarrolla actividades pedagógicas en Educación No Formal respondió la pregunta: ¿En sus actividades o ejercicios pedagógicos empleas componentes artísticos? "Por supuesto, dado que las distintas actividades pedagógicas que he realizado han sido, en su mayoría, enfocadas a la iniciación dentro de un lenguaje artístico, (en mi caso, la técnica vocal) y teniendo en cuenta que tales clases se han dado dentro del movimiento de educación popular y promoción social Fe y Alegría, y siendo fiel a los ideales que éste tiene para con el ser, me he valido de las fortalezas que el arte ofrece para con la sensibilización de la persona con el fin de impulsar y despertar habilidades como la creatividad y la expresión llevadas a su máximo esplendor, a través de alguna de las disciplinas artísticas, en este caso, la música".

El líder de las prácticas pedagógicas del Programa, que a su vez es egresado de la misma Licenciatura, respondió a la siguiente pregunta: ¿Cómo estructura o diseña esos componentes artísticos? "En mi caso no requieren un planteamiento de especial atención. Mi trabajo es básicamente disciplinar, lo que implica una comunicación asertiva y efectiva sobre el hacer. Por otra parte, la demostración y compartir inquietudes para resolver en clase, despoja de misterios la practica disciplinar y las dificultades que se presenten se abordan desde los recursos pedagógicos, lo que facilita las soluciones propuestas" (Winston Sánchez, comunicación personal, 12 de septiembre de 2016).

Con estas dos participaciones, se entiende que el concepto de arte está basado en el desarrollo disciplinar, es decir, la educación artística y, en este caso, la enseñanza de las artes debe trasmitir unos conocimientos específicos sobre técnicas con el fin de lograr la elaboración precisa de determinadas propuestas artísticas.

Por otra parte, la Directora del Área de Humanidades, evidencia ciertas expectativas del arte que amplía el campo de acción de la educación artística, a través de la respuesta que proporcionó a la pregunta: ¿Qué crees que se relaciona con todo esto y esa necesidad de resolver problemas de las sociedades con un licenciado en educación artística? " Mira que yo siempre he visto el arte como una manera de aprender, es la 
forma de llegar a las cosas, es como un intermediario mágico para entender ciertas cosas. Además, creo que es poderosísimo, tiene mucho poder para mí. El arte puede ser un comunicador entre la persona y la realidad, puede ser una manera de conectar, entonces allí el papel de mediación que tiene el arte es poderosísimo, creo que la Universidad debería aprovecharlo más, que ustedes tuvieran más eco en todos los procesos de la Universidad [...] Pues yo creo que en su ejercicio sí, digamos que manteniendo el ejercicio se puede convertir en el mediador a través de la educación, digamos que es el principio, como cuando uno piensa en el perfil profesional de un abogado, como yo pensar en el perfil profesional, de un licenciado que es la educación, entonces educar a través del arte me parece que es un insumo para Unicatólica, para acercarse a cualquier realidad" (Valentina Betancourt, comunicación personal, Agosto 18 de 2016).

Esta respuesta permite comprender que el arte no solo debe ser el resultado perteneciente a las diferentes disciplinas artísticas, sino que estas deben entenderse como un detonador de procesos reflexivos y críticos, al igual que el concepto que enmarca de la educación artística, pues deberá "educar a través del arte", es decir, que el arte debe contribuir a la formación de un ciudadano perteneciente a una sociedad, lo que muy probablemente hace que la propuesta disciplinar tenga objetivos diferentes a la presentación de obras artísticas.

\section{Conclusiones y recomendaciones}

El proyecto evidenció las fortalezas que tiene la Licenciatura, a través del ejercicio que sus estudiantes desarrollan en la Educación No Formal y del conocimiento que este espacio les ha proporcionado en educación y pedagogía social que, aunque no haya una coherencia completa entre el discurso y la práctica, es importante señalar que muchas de las intenciones que dirigen el discurso, implican una apuesta por comprender al sujeto como un ser en construcción por medio del ejercicio dialógico entre este y su docente o estudiante, y de este con el entorno que lo contextualiza. Es decir, los estudiantes comprenden que el sujeto hace parte activa de una sociedad.

También evidenció que desde LEBEA es necesario buscar las estrategias que le permitan a los estudiantes articular el ejercicio práctico a su discurso. Es decir, que puedan demostrar en sus actos la coherencia entre el concepto de sujeto activo dentro de una sociedad y la revisión del contexto local, nacional o global en el que se enmarcan estos sujetos y la participación que ha este se le permita en la construcción del hacer educativo; entre el concepto de arte y la práctica pedagógica de la educación artística; además de este mismo concepto con los ideales de educación y, por ende, la debida articulación de la educación artística a estos dos.

En este sentido, el Proyecto pone en relieve una problemática que se gesta desde las definiciones de educación artística a nivel gubernamental y que involucra sus funciones dentro de la Educación Básica y Media emitidas por las instituciones que prospectan el campo de conocimiento a nivel global. Puesto que, en términos generales, según la UNESCO, la tarea de la educación es la construcción de ciudadanos. Ante esta tarea cabe preguntar por la forma como debe de articularse la educación artística, qué significaría para la educación artística construir ciudadanos, de qué manera la enseñanza de las disciplinas artísticas contribuyen a este objetivo, qué prácticas desde las artes contemporáneas aportan a este objetivo, cómo se lograría llevar a la Educación Básica y Media estos procesos artísticos, qué discusiones se suscitarían al respecto si la educación artística se enfocara más a la construcción de un ciudadano a través del ejercicio artístico, alejándose de la tradicional propuesta en la que esta debe formar disciplinariamente en habilidades y capacidades artísticas a los estudiantes.

\section{Referencias bibliográficas}

Betancourth, V (2016, agosto 18). Comunicación Personal

Buzzi, A. (2016, Agosto 22). Comunicación Personal

Cambi, F. (2005) Las pedagogías del Siglo XX. Madrid: Popular.

Freire, P. (2002) Pedagogía del oprimido. Buenos Aires, Siglo XXI.García Molina, J. L. (2003) Dar (la) palabra: deseo, don ética en educación social. Barcelona: Gedisa.

Garcia, G. (2016 Octubre 10). Comunicación Personal

Garcia, J. (2003). Dar la Palabra, Barcelona. Gedisa 
Giroux, H. A. (2001) Cultura, política y práctica educativa. Barcelona: Gráo.

Gouldner, A. (1978). El discurso ideológico como racionalidad y falsa conciencia. En La dialéctica de la ideología y la tecnología. Madrid: Alianza Universidad (Pp. 47-97).
Quiceno, P. (2016, Agosto 22). Comunicación Personal

Rubio, J. (2015) Educación y Pedagogía Social. Ciudad: Sé

Sánchez, W. (2016, Septiembre 12). Comunicación Personal. 